\title{
Photonic chip-based multimodal super-resolution microscopy for histopathological assessment of cryopreserved tissue sections
}

\author{
Luis E. Villegas-Hernández
}

The Arctic University of Norway

\section{Vishesh Dubey}

The Arctic University of Norway https://orcid.org/0000-0002-2753-0445

\section{Mona Nystad}

Department of Obstetrics and Gynecology, University Hospital of North Norway

Jean-Claude Tinguely

The Arctic University of Norway

\section{David A. Coucheron}

The Arctic University of Norway

\section{Firehun T. Dullo}

The Arctic University of Norway

\section{Anish Priyadarshi}

The Arctic University of Norway

\section{Sebastian Acuña}

The Arctic University of Norway

\section{Jose M. Mateos}

Center for Microscopy and Image Analysis, University of Zurich,

\section{Gery Barmettler}

Center for Microscopy and Image Analysis, University of Zurich,

\section{Urs Ziegler}

Center for Microscopy and Image Analysis, University of Zurich,

\section{Aud-Malin Karlsson Hovd}

Department of Medical Biology, RNA and Molecular Pathology Research Group, UiT The Arctic University of Norway

\section{Kristin Andreassen Fenton}

Department of Medical Biology, RNA and Molecular Pathology Research Group, UiT The Arctic University of Norway

\section{Ganesh Acharya}

Karolinska University Hospital 
The Arctic University of Norway

\section{Balpreet Singh Ahluwalia ( $\square$ balpreet.singh.ahluwalia@uit.no)}

The Arctic University of Norway https://orcid.org/0000-0001-7841-6952

\section{Article}

Keywords: microscopy, photonic chips, histopathological assessment

Posted Date: May 10th, 2021

DOI: https://doi.org/10.21203/rs.3.rs-500460/v1

License: (1) This work is licensed under a Creative Commons Attribution 4.0 International License. Read Full License 


\section{Abstract}

Histopathological assessment involves the identification of anatomical variations in tissues that are associated with diseases. While diffraction-limited optical microscopes assist in the diagnosis of a wide variety of pathologies, their resolving capabilities are insufficient to visualize some anomalies at subcellular level. Although a novel set of super-resolution optical microscopy techniques can fulfill the resolution demands in such cases, the system complexity, high operating cost, lack of multimodality, and low-throughput imaging of these methods limit their wide adoption in clinical settings. In this study, we interrogate the photonic chip as an attractive high-throughput super-resolution microscopy platform for histopathology. Using cryopreserved ultrathin tissue sections of human placenta, mouse kidney, and zebrafish eye retina prepared by the Tokuyasu method, we validate the photonic chip as a multi-modal imaging tool for histo-anatomical analysis. We demonstrate that photonic-chip platform can deliver multi-modal imaging capabilities such as total internal reflection fluorescence microscopy, intensity fluctuation-based optical nanoscopy, single-molecule localization microscopy, and correlative lightelectron microscopy. Our results demonstrate that the photonic chip-based super-resolution microscopy platform has the potential to deliver high-throughput multimodal histopathological analysis of cryopreserved tissue samples.

\section{Introduction}

Histopathology refers to the study of tissue sections under a microscope to diagnose diseases, guide medical treatment, and prognose clinical outcomes. To date, this well-established discipline is one of the key decision-support tools available for clinicians across the world. A typical histological analysis involves the extraction of a tissue sample from the body, fixation, and preservation followed by sectioning, labeling, and microscopy. By performing a morphological assessment of the tissue under the microscope, histopathologists can identify various diseases and render a clinical diagnosis.

Imaging throughput, contrast, and resolution are critical parameters in the histopathological assessment. Given the morphological heterogeneity of the samples, pathologists often need to assess tens to hundreds of $\mathrm{cm}^{2}$ section areas to locate and analyze the lesions [1]. Thus, high-throughput imaging platforms are desirable for routine histopathological analysis. The whole slide imaging scanners fulfill this requirement by allowing fast imaging of several histological slides in a day. However, these automated optical microscopes are limited to a resolution power of |tilde 250-500 nm [2], which is insufficient for the identification of some pathologies, for example, nephrotic syndrome and amyloidosis. For decades, the visualization of such pathologies was only possible through other imaging techniques such as electron microscopy [3, 4], which supports a resolving power down to Itilde $10 \mathrm{~nm}$ for fixed and embedded histological samples. However, the combination of a lengthy sample preparation process, a low imaging throughput, and the lack of specificity makes electron microscopy an inconvenient and costly technique for clinical use, hindering its broad adoption for routine histopathological examination of tissue samples and restricting its implementation to basic-biology research. 
Recently, the advent of super-resolution fluorescence optical microscopy techniques, also referred to as optical nanoscopy, bridged the resolution gap between the diffraction-limited optical microscopy and the electron microscopy methods, allowing for high-specificity imaging of biological specimens at highresolution [5-7]. Present-day fluorescence-based super-resolution optical microscopy comprises a panel of methods that exploit engineered illumination and/or the photochemical and photokinetic properties of fluorescent markers to achieve high spatio-temporal resolution. These include structured illumination microscopy (SIM), stimulated emission depletion microscopy (STED), single-molecule localization microscopy (SMLM), and intensity fluctuation-based optical nanoscopy techniques (IFON).

While super-resolution fluorescence optical microscopy methods are commonly used in cell biology, their adoption in histopathological settings remains deferred due to multiple reasons: a) the high labelling density of tissues poses challenges on super-resolution methods, especially for SMLM and IFON, where a high spatio-temporal sparsity is necessary for the reconstruction of structures beyond the diffraction limit of the microscope; $b$ ) the susceptibility of the super-resolution methods to optical aberrations and light scattering introduced by refractive index variations across the samples [8]; c) the imaging artifacts induced by autofluorescence signal of tissues [9]; and importantly, d) the low throughput, high-cost, lack of multi-modality, system complexity, and bulkiness of existing super-resolution optical microscopy setups.

Although, limited work on using STED, SIM, and SMLM have been explored for super-resolution imaging of tissues [10-12], these methods fail to fulfill the throughput demands for routine histopathological assessment (see Supplementary Information S1). For example, STED, albeit delivering a lateral resolution down to $20 \mathrm{~nm}$ [13] and being a robust confocal method to scattering challenges posed by tissues, is an inherently low-throughput point-scanning technique. Similarly, SMLM and SIM, despite being wide-field methods supporting sub-50 nm and Itilde 110-130 nm lateral resolution respectively, are heavily dependent on the acquisition of multiple frames and subsequent reconstruction via post-processing algorithms. While SIM outperforms SMLM in terms of imaging speed, requiring only 9 or 15 images (2D/3D cases accordingly) as compared to the tens of thousands of images necessary for SMLM, the field of view obtained by commercial SIM systems is typically limited to about $40 \times 40 \mu \mathrm{m}^{2}$. Importantly, among all the super-resolution methods, SIM has been proposed for high throughput imaging in histopathological settings $[1,11]$. However, these approaches focused on the acquisition of large field of view images using low magnification and low numerical aperture objective lenses, compromising the lateral resolution to a maximum of $1.3 \mu \mathrm{m}$. In terms of system complexity, SMLM is simpler to implement as compared to SIM and STED, which require more sophisticated, bulkier, and costly setups. From an overall perspective, improvements in imaging throughput and reductions in system complexity, footprint, and cost are needed for the adoption of super-resolution fluorescence optical microscopy in histopathology. It is evident from Supplementary Table S1 that different imaging methods offer different technical capabilities. Thus, to enable widescale penetration in the clinical settings, it is desirable to have an imaging platform that can deliver different super-resolution capabilities using standard optical microscopy setup. 
Another important aspect for the adoption of fluorescence-based super-resolution optical microscopy is the availability of a large selection of fluorophores. While SIM works with photo-stable and bright fluorophores, STED and SMLM are more restricted to a special type of fluorescent markers. Interestingly, some of the IFON techniques, such as the multiple signal classification algorithm (MUSICAL) [14], can exploit the pixel intensity variations arising not only from the intrinsic fluctuations of the fluorophores but also from the modulated emissions generated via engineered illumination, enabling a practical implementation with almost all kinds of fluorophores. Despite being an attractive route to follow for clinical applications in histopathology, to the best of our knowledge the engineered illumination approach for IFON has not been explored in tissue imaging.

In recent years, photonic chip-based nanoscopy has emerged as a promising imaging platform for biological applications [15-18], supporting high-resolution, high-throughput, and multi-modal capabilities. To date, photonic chip-based microscopy studies have focused primarily on cellular biology [15-21], leaving on-chip histological imaging relatively unexplored. In this work, we interrogate the photonic chip-based imaging platform to address some of the challenges related to super-resolution imaging of tissue sections. We start by evaluating the viability of the photonic chip for diffraction-limited total internal reflection microscopy (chip-TIRFM). Then, we transition to more advanced chip-TIRFM based imaging methods such as SMLM, IFON, to conclude with a correlative light-electron microscopy (CLEM) analysis. Among the existing histological methods, we chose the Tokuyasu protocol [22] for the preparation of the tissue sections. This cryosectioning method provides excellent ultrastructural preservation, high molecular antigenicity, and a thin section thickness ( $70 \mathrm{~nm}$ to $1 \mu \mathrm{m})$ that assists both in reducing the light scattering artifacts associated with thicker samples [23] and in making optimal use of the illumination delivered by the photonic chip. We describe the staining protocols and the imaging parameters necessary for photonic chip-based microscopy of tissue samples and discuss the challenges and the advantages offered by this imaging platform for histopathology. By exploiting the engineered illumination delivered by the photonic chip-based microscopy, we further demonstrate the suitability of this novel technique as a compact, high-resolution, high-contrast, high-throughput, and multi-modal imaging platform for histopathology.

\section{Photonic Chip-based Microscopy For Histopathology}

In chip-based microscopy, a photonic chip is used both to hold the sample and to provide the excitation illumination necessary for fluorescent emission (Fig. 1), while a standard upright microscope is used to acquire the image (Fig. 1c). The photonic chip is composed of two substrate layers of silicon (Si) and silicon dioxide $\left(\mathrm{SiO}_{2}\right)$, respectively, and a biocompatible waveguide core layer that transmits visible light, made of either silicon nitride $\left(\mathrm{Si}_{3} \mathrm{~N}_{4}\right)$ [17] or tantalum pentoxide $\left(\mathrm{Ta}_{2} \mathrm{O}_{5}\right)$ [24]. Upon coupling, the excitation laser beam is tightly confined inside the optical waveguide layer and propagates through its geometry via total internal reflection (Fig. 1a). This generates an evanescent field on the top of the waveguide surface with a penetration depth of up to Itilde 150-200 nm that is used to excite the fluorescent markers located in the vicinitv of the waveguide surface (see Supplementary Information S2). Loading [MathJax]/jax/output/CommonHTML/jax.js 
The fluorescent emission is then collected by a standard microscope objective, enabling chip-TIRFM (Fig. 1e).

Photonic chip-based illumination provides several advantages that can be exploited for super-resolution imaging of histopathology samples such as:

a)The photonic chip allows decoupling of the excitation and the emission light paths, which translates into high-contrast images with improved imaging throughput. The propagating light enables a uniform illumination over the entire length of the waveguide while providing optical sectioning of the sample via evanescent field excitation [25]. As the illumination is provided by the photonic chip, the imaging objective lens can be freely changed (Fig. 1d), enabling the acquisition of images over large fields of view [16] (Fig. 1e), a feature not available in conventional TIRFM setups.I

b) The multi-mode interference illumination generated on the photonic chip assists in generating the necessary emission sparsity for diverse super-resolution fluorescent optical microscopy methods, as recently demonstrated via on-chip IFON [15, 26], on-chip SMLM [15, 16, 27], and on-chip SIM [28].

Moreover, by using waveguide materials of high refractive index (for example, $n=2$ ), it is possible both to tightly confine the light and to generate higher spatial frequencies as compared to free-space optical components $[15,28]$, which can be further exploited by IFON techniques such as MUSICAL to superresolve highly dense and heterogeneous samples such as tissues.

c) Correlative imaging with other established methods including electron microscopy [29] and quantitative phase microscopy [24] can be seamlessly implemented on the photonic chip, expanding the opportunities both for routine analysis and for basic histopathology research.

d) The photonic chip-based microscopy can be implemented on standard optical microscopy platforms upon few adaptations for the integration of a photonic chip module (Fig. 1C). The photonic chips can be manufactured in high-volumes following standard complementary metal-oxide-semiconductor (CMOS) photolithography processes, allowing for low operating costs in clinical settings.

\section{Results And Discussion}

\subsection{Chip-based multicolor TIRFM imaging}

In this part of the study, we used chorionic villi tissue from human placenta to assess the suitability of the photonic chip for histological observations. This tissue, present on the fetal side of the placenta, is responsible for the air, nutrient, and waste exchange between the mother and the fetus during pregnancy [30], and is characterized by villous-like structures, namely villi, that sprout from the chorionic plate of the placenta to maximize the maternofetal transfer processes and communication. When transversally sectioned, the chorionic villi appear in the form of rounded islands distributed across an open space surrounded by maternal blood, called the intervillous space. 
Developed by Kiyoteru Tokuyasu in the '70s [31, 32], the so-called "Tokuyasu method" is still a gold standard protocol for ultrastructural analysis of cells and tissues [22]. Primarily established for EM techniques, recent studies have shown its versatility in fluorescence microscopy [33, 34]. For chip-based multicolor TIRFM imaging, $400 \mathrm{~nm}$ thick chorionic villi cryosections were prepared following the Tokuyasu method (see detailed preparation protocol in Materials and Methods and Supplementary Information S3). After cutting the tissue blocks on a cryo-ultramicrotome, the sections were deposited onto a photonic chip previously coated with poly-L-lysine and equipped with a custom-made transparent polydimethylsiloxane (PDMS) frame (Fig. 1b). The membranes, F-actin, and nuclei were fluorescently labeled using CellMask Deep Red, Phalloidin-Atto565, and Sytox Green, respectively.

For the excitation of the respective fluorescent dyes, three independent laser light wavelengths were used, namely $640 \mathrm{~nm}, 561 \mathrm{~nm}$, and $488 \mathrm{~nm}$ (Fig. 1d). To obtain TIRF images (see detailed acquisition steps in Materials and Methods), the excitation light was coupled onto a single strip waveguide using a 50X/0.5NA microscope objective (Fig. 1c). Upon coupling, a multi-mode interference pattern was generated along the waveguide by the propagating light, which could be modulated by changing the position of the coupling objective relative to the chip (see Supplementary Information S4). To deliver a uniform illumination onto the sample, the coupling objective was laterally scanned along the input facet of the chip while individual frames were acquired. The fluorescent emission was collected by standard microscope objectives transitioning from lower to higher magnification to achieve different fields of view. Thereafter, the collected signal was averaged, pseudo-colored (membranes in red, F-actin in green, and nuclei in blue), and merged, allowing multicolor visualization of the different tissue components.

The large field of view provided by the $4 \mathrm{X} / 0.1 \mathrm{NA}$ objective lens enabled us to locate the sample on the waveguide (Fig. 2a), while the 20X/0.45NA assisted for a contextual visualization of the tissue structure, supporting the identification of regions of interest for imaging with further magnification (white box in Fig. 2b). Finally, with the aid of a 60X/1.2NA water immersion objective lens (Fig. 2c), it was possible to visualize relevant structures of the chorionic villi, such as the apical layer of syncytiotrophoblastic cells, and the abundant fetal capillaries. Arguably, in this study, the absence of maternal red blood cells in the intervillous space can be attributed to the rinsing steps carried out along with the sample collection (see Materials and Methods). Figure $2 \mathrm{c}$ also allows the visualization of multinucleated cell aggregates that resemble the syncytial knots usually deported onto the maternal blood at different stages of the pregnancy [35]. Notably, the membrane marker not only allowed for an overall view of the tissue (Fig. 2b,c) but also enabled the distinction between adjacent cells such as a cytotrophoblast cell and a syncytiotrophoblast cell (white box in Fig. $2 \mathrm{c}$ and magnified view in Fig. 2d). Moreover, the observed Factin signal (Fig. 2e) matched the locations reported in a previous study [36], allowing the identification of the microvilli brush border, the syncytiotrophoblastic's basal cell surface, and the capillary endothelial cells.

The cross-sectional dimensions of the Tokuyasu sections (typically ranging between $300 \times 300 \mu \mathrm{m}^{2}$ and $500 \times 500 \mu^{2}$ ) perfectly suited the waveguide dimensions of the photonic chip used in this work. This Loading [MathJax]/jax/output/CommonHTML/jax.js of the sample through a single optical waveguide and also 
supports independent illumination of adjacent waveguides on the chip with different tissue sections (Fig. 1b). This eliminates undesired excitation light of the samples outside the imaging region of interest, hence minimizing photobleaching. Moreover, the PDMS chambers (Fig. 1b) allowed multi-well experiments similarly to traditional microscope chamber slides, with the additional advantage of reducing the incubation volumes to approximately $10 \mu \mathrm{l}$ to $20 \mu \mathrm{l}$ per chamber, which translated into a costreduction of the fluorescence assays. After optimizing the sample preparation and imaging steps (see Supplementary Information S5, S6, and S7), we were able to both fluorescently label and acquire chipTIRFM images of placental tissue within a timeframe of three hours from cryosectioning to image postprocessing.

For diffraction-limited imaging of tissue samples, such as shown in Fig. 2, the evanescent field illumination supported by TIRFM is not necessary. However, for super-resolution methods such as SMLM and IFON, the evanescent illumination generated by the photonic chip configuration plays a key role in supporting optical sectioning of the specimen, reducing the out-of-focus light, increasing the signal-tobackground ratio, and improving the axial resolution. Conventional TIRFM setups use oil-immersion high numerical aperture (N.A. 1.47-1.50) and high-magnification objective lenses (60X - 100X) that limit their field of view to around $50 \times 50 \mu \mathrm{m}^{2}$ [20] (dotted box in Fig. 2b). On contrary, the photonic chip-based TIRFM setup allows the use of essentially any imaging objective lens for the collection of the fluorescent signal, achieving scalable resolution and magnification on demand and opening possibilities for large TIRFM imaging areas up to the $\mathrm{mm}^{2}$ scale (Fig. 2a, b). To this extend, the photonic chip-based TIRFM technique has the potential to outperform traditional ways of generating an evanescent field, which can be exploited for super-resolution imaging, as detailed in the next sections.

\subsection{Chip-based SMLM imaging}

In the previous section, we demonstrated the suitability of the photonic chip for diffraction-limited TIRFM imaging of tissues. Here, we explored on-chip super-resolution imaging of tissue samples using singlemolecule localization microscopy (SMLM) [5]. SMLM comprises a set of methods that exploit the stochastic activation of individual fluorescent molecules to enable their precise localization within a subdiffraction limited region. To achieve this, the fluorescent molecules are manipulated to obtain sparse blinking events over time. In practice, the majority of the fluorophores are switched off (not emitting light), while only a small segment of them is switched on (emitting fluorescence). This implies the collection of several thousands of frames for the localization of the individual molecules in the sample.

There exist multiple variants of SMLM employing diverse switching mechanisms. Among them, the direct stochastic optical reconstruction microscopy (dSTORM) method supports conventional fluorophores, delivers a high photo-switching rate, and offers low photobleaching [37]. To explore the capabilities of the photonic chip for SMLM on histological samples, we used a $400 \mathrm{~nm}$ thick mouse kidney cryosection. We employed a dSTORM approach to visualize the ultrastructural morphology of the filtration compartments present in the renal tissue, called glomeruli, whose physical dimensions are typically beyond the resolution limit of conventional optical microscopy and, therefore, often studied through electron 
The membranes and the nuclei were fluorescently stained with CellMask Deep Red and Sytox Green, respectively. All the preparation steps were performed identically to the chip-based multicolor TIRFM imaging experiments, except for the mounting medium that consisted of a water-based enzymatic oxygen scavenging system buffer $[15,16]$ (see details in Supplementary Information S8). This oxygen scavenging buffer induces the blinking behavior by enhancing the probability of the fluorescent molecules to transition into the dark state, thereby contributing to the temporal sparsity of emission necessary for SMLM.

To find the features of interest, a TIRFM image of the sample was acquired (Fig. 3a) using low laser power to avoid photo-switching and reduce the chances of photo-bleaching. Next, the laser power was increased until sparse blinking was observed. The camera exposure time was set to around $30 \mathrm{~ms}$ to capture individual emission events of the membrane dye while the coupling objective was randomly scanned along the input facet of the chip. The collected image stack ( $>40,000$ frames) was computationally processed to localize the spatial coordinates of the fluorophores, allowing for the reconstruction of a super-resolved image (Fig. 3b). A comparative view of both methods (Fig. 3c) reveals structural details in dSTORM that are not discernible in diffraction-limited TIRFM. In particular, dSTORM allows the visualization of a Itilde $100 \mathrm{~nm}$ gap between the podocytes and the endothelial cells (see the empty gap between the white arrows in Fig. 3c), which is in agreement with the morphology of the glomerular basal membrane [38]. The identification of this feature, in particular, may be of critical value for a faster diagnosis of nephrotic diseases.

Chip-based SMLM/ dSTORM supports three to four-fold resolution improvement over diffraction-limited imaging using a standard upright optical microscopy set-up with a slight modification. Moreover, the chip-based SMLM/ dSTORM approach benefits from the inherent advantage of decoupled illumination and collection light paths, which allows a user-defined choice of imaging objective lens without altering the TIRF excitation delivered by the chip. With further efforts in immunolabeling (see Supplementary Information S9) and system automation, chip-based SMLM could dramatically shorten the diagnostic time of nephrotic diseases that, up to now, are identified via low-throughput and expensive methods such as electron microscopy. While chip-based illumination enables the imaging of large areas, the essential challenge of SMLM relies on the need for a large number of frames for the reconstruction of a superresolved image. Therefore, for routine histopathological analysis, it is opportune to explore alternative imaging methods, e.g. IFON, with lower demands in the number of frames necessary for super-resolution.

\subsection{Chip-based IFON imaging}

To achieve a shorter acquisition time while maintaining imaging of large areas with improved contrast and resolution, we explored chip-based intensity fluctuation optical nanoscopy (IFON) of tissue samples. IFON comprises a set of techniques that exploit the photokinetic properties of fluorescent molecules to resolve structures beyond the diffraction limit of optical microscopes [39]. The techniques examine the stochastic emission of fluorophores through statistical analysis of the intensity levels of a given image stack, allowing the identification of fluorescent emitters with sub-pixel precision. Among the IFON 
fast and reliable image reconstruction of biological data [39], achieving sub-diffraction resolution through low excitation intensities, fast acquisition, and relatively small datasets (100-1000 frames per image stack).

The main challenges to implement IFON on histological samples are the high density and heterogeneity of the tissue samples. The spatio-temporal fluctuations are a decreasing function of the spatial density of the labels. In other words, a high density of labels results in a higher average signal at the cost of low variance in the fluorescence intensity over time. As a consequence, typically the IFON techniques are demonstrated on fine sub-cellular structures (e.g. actin filaments, microtubules, and mitochondria) fluorescently labeled on plated cells. Thus, densely labeled structures such as endoplasmic reticulum or lipid membranes are generally avoided. Tissue samples, with a higher density of labels, put even stronger demands on computational algorithms. Here, instead of relying only on the intrinsic fluctuations of the fluorophores, we propose to exploit also the intensity variations induced by the multi-mode interference (MMI) pattern (speckle-like illumination) generated by the photonic chip (see Supplementary Information S4). In this approach, on-chip MMI illumination patterns are modulated over time by scanning the illumination spot over the waveguide input facet. This modulates the fluorescence emissions from the fluorophores with the spatial intensity distribution of the illumination pattern at any given time. Due to the constructive and destructive interferences, bright and dark regions are formed, artificially introducing sparsity in the spatiotemporal fluctuations. In addition, due to the high refractive index of the waveguide core $\left(n=2.1\right.$ for $\mathrm{Ta}_{2} \mathrm{O}_{5}$ and $n=2$ for $\left.\mathrm{Si}_{3} \mathrm{~N}_{4}\right)$, the MMl pattern obtained on top of the waveguide surface are sub-diffraction limit and thus carry higher spatial frequencies than what can be obtained using freespace optics [15]. Here, we used such on-chip engineered illumination for super-resolution imaging using the MUSICAL method.

To interrogate the capability of the photonic chip for IFON-based imaging of histological samples, we used chorionic villi tissue cryosections from human placenta. For IFON studies, we focused on the visualization of ultrastructural features in the microvilli. The microvilli are actin-based membrane protrusions that increase the contact area between the syncytiotrophoblastic cells and the maternal blood, facilitating the biochemical exchange between the maternal and the fetal side, and supporting mechano-sensorial functions of the placenta [40]. Due to the physical dimensions of these structures (on average, $100 \mathrm{~nm}$ in diameter and $500 \mathrm{~nm}$ in length [41, 42]), and their tight confinement along the apical side of the syncytiotrophoblastic cells, the morphological features of the microvilli are not discernible through conventional optical microscopy and, therefore, represent an ideal element to benchmark the resolution possibilities offered by chip-based IFON.

The samples were prepared and imaged with a 60X/1.2NA microscope objective following the steps described for Chip-based multicolor TIRFM imaging. To avoid unspecific background signal, only the Factin and nuclei markers were used (Phalloidin-Atto565 and Sytox Green, respectively). Further, the 500frames image stack corresponding to the F-actin was analyzed with MUSICAL, resulting in a superresolved and improved contrast image over a field of view of $220 \times 220 \mu \mathrm{m}^{2}$ (Fig. 4a). The Loading [MathJax]/jax/output/CommonHTML/jax.js ne in MUSICAL [43] allowed the identification of individual 
microvilli along the syncytiotrophoblast's brush border (Fig. 4d), which were otherwise unclear in the averaged chip-TIRFM image (Fig. 4b). The resolution enhancement of MUSICAL is quantified through line-profile measurements over two adjacent microvilli. Where chip-TIRFM image (Fig. 4c) showed two structures merged as a single element, the MUSICAL reconstruction (Fig. 4e) revealed the separation between them. On-chip MUSICAL not only increases the resolution but improves the contrast of the image, which is a valuable parameter during visual investigations by histopathologists.

A recent study reported the visualization of individual microvilli with a 2-fold resolution improvement employing 3D-SIM [44]. Although several experts have proposed SIM as the fastest SRM technique for histopathology $[1,11,45,46]$, the typical FOV of this method with high magnification microscope objectives (for example, 60X/1.42NA) is about $40 \times 40 \mu \mathrm{m}^{2}$. Therefore, to match the same field of view achieved with the photonic chip, a tile mosaic of $7 \times 7$ SIM images would be required (see Supplementary Information S10). For conventional 3D-SIM, this not only implies a prolonged time for the data acquisition, but also a lengthy image reconstruction that rounds up to $2.5 \mathrm{~h}$. On contrary, the MUSICAL implementation we used here was able to obtain a high-resolution image over a large field of view within a combined collection and processing time of $\backslash$ tilde $10 \mathrm{~min}$ for the 500 -frames acquired on the photonic chip. From a practical perspective, the high-resolution visualization over large areas supported by chipbased IFON opens the door for improved assessment of placental microstructure both for basic research as well as for clinical assessment of placental pathologies associated with morphological changes in the microvilli, as documented in placental dysfunction disorders, such as pre-eclampsia [41].

\subsection{Chip-based CLEM imaging}

Combining the specificity of fluorescence microscopy with the high resolution of electron microscopy allows the visualization of proteins of interest along with the ultrastructural context of the tissues. Although recent reports have proposed silicon wafers for correlative light and electron microscopy (CLEM) [47, 48], they employed EPI-illumination through high-magnification microscope objectives, providing a limited field of view of the fluorescent signal. Here, we employed zebrafish eye retina cryosections of $110 \mathrm{~nm}$ thickness to demonstrate the compatibility of the chip platform with CLEM studies. Zebrafish is a well-established model for the study of retinal diseases [49]. The samples were prepared in the same manner as the placental and renal sections, except for the initial washing steps of the cryoprotectant. We found that the optimal washing temperature of the sucrose-methyl cellulose solution for these samples was $0^{\circ} \mathrm{C}$, over an incubation time of 20 minutes (see detailed protocol in Materials and Methods). Three structures were labeled for the study: a) the F-actin filaments, $b$ ) the nuclei, and c) the outer mitochondrial membrane. The first two structures were labeled through direct markers using Texas Red-X Phalloidin and Sytox Green, respectively, whereas the latter was labeled by immunofluorescence using rabbit anti-Tomm20 as a primary antibody, and Alexa Fluor 647-conjugated donkey anti-rabbit as a secondary antibody.

The samples were first imaged in chip-based TIRFM mode for each channel using a 60X/1.2NA to obtain a diffraction-limited multicolor image (Fig. 5a). Thereafter, the sections were platinum-coated and imaged 
TIRFM image (Fig. 5c) allows for the observation of the F-actin filaments lining the outer segments of the photoreceptors (in green), as well as the mitochondria clusters (in magenta), and the location of the nuclei (in cyan). The same TIRFM dataset is used for post-processing through MUSICAL, allows for a precise correlation of both the F-actin and the mitochondrial signals with the corresponding SEM image (Fig. 5d,e,f).

Notably, the waveguide widths on the chip not only accommodated the whole zebrafish retina but also allowed the observation of several serial sections in a ribbon (see Supplementary information S12). Also, the combination of the thin section thickness of the Tokuyasu samples with the limited extent of the evanescent field dramatically improved the axial resolution of the fluorescent signal, enabling highcontrast images. Put together, these features are advantageous for confirming signal specificity throughout different subcellular compartments, opening up the possibility for 3D-stacking via serial section imaging $[50,51]$. Moreover, the flatness of the chip serves as an optimal platform for SEM, allowing autonomous imaging over large areas. A simple thin layer of platinum deposited on top of the chip minimizes the charging effects and enables a good correlation between the light and the electron microscopy images. Importantly, the photonic chip can incorporate coordinate land-markings to facilitate the location and further correlation of the ROIs under study [29]. Lastly, the chip-based CLEM strategy presented here, in combination with the Tokuyasu method, can be executed within one working day from the sample sectioning steps to the SEM imaging, implying a significant time improvement as compared to the typical one-week imaging throughput associated with most CLEM approaches [34].

\section{Conclusions And Future Perspectives}

In this study, we demonstrated the capabilities of the photonic chip as a feasible imaging platform for morphological assessment of thin Tokuyasu sections of a variety of tissues. The photonic chip-based microscopy technique offers several advantages for histopathology: a) it allows a broad range of imaging modalities over large fields of view including TIRFM, SMLM, IFON, and CLEM using a single standard optical microscope set-up; b) the imaging process can be seamlessly performed on conventional optical microscopes upon some modifications; and c) the photonic chip withstands all the chemical incubations and thermal conditions associated with the sample preparation. These features make the photonic chip an attractive platform for fluorescence-based histopathological studies where high-throughput, high-contrast, and high-resolution are crucial for the diagnosis of diseases. We anticipate that, upon specific labeling and image processing efforts, the photonic chip could assist both in reducing the processing time and in improving the assessment quality of pathologies that -to daterequire ultrastructural observation using electron microscopy. Additionally, in CLEM experiments, the photonic chip could be used for fast assessment of ultrastructural preservation in tissues.

The photonic chip approach also reduces the complexity of the optical nanoscopy setups by miniaturization of the excitation light path, simplifying the implementation of multimodal imaging and facilitating a larger adoption of super-resolution microscopy in clinics and hospitals. In addition, the 
from low-cost manufacturing scalability. We foresee that further developments in coupling automation and the integration of microfluidics systems could dramatically improve the performance of the photonic chip platform, enabling more efficient and repeatable labeling, as well as fast multiplexed imaging. Moreover, the implementation of advanced labeling strategies such as DNA-PAINT [27] and ExchangePAINT [52] can effectively reduce the background signal, improving resolution, and support multiplexed acquisition. Also, on-chip technology facilitates the integration of other on-chip optical functions such as Raman spectroscopy [53], waveguide trapping [54], microfluidics [55], phase microscopy [24], among others.

While the photonic chip illumination strategy allows excitation over large areas, e.g. several centimeters in the present case, the light collection area is presently limited by the collection objective lens. Thus, it can be envisioned that the integration of microlens arrays [56] for light collection will open avenues that would make on-chip technology capable of handling the high-throughput imaging needed for routine histopathology. Moreover, the photonic chip can be designed and manufactured into standard microscope glass slide dimensions, allowing for a fully automated sample preparation through commercially available immunoassay analyzers, or via novel microfluidic techniques for multiplex immunofluorescence staining of clinically relevant biomarkers [57].

Despite the encouraging imaging results obtained in this study, we acknowledge that the Tokuyasu samples represent a minority among the available histological methods. Also, we are aware that the maximum section area possible with the Tokuyasu cryosections $\left(500 \times 500 \mu \mathrm{m}^{2}\right)$ may be insufficient for large-scale histopathological evaluation. However, this is an inherent limitation imposed by the sample preparation technique rather than the photonic chip imaging surface. Future chip-based histology studies should address the compatibility of this microscopy platform with widely accessible samples including formalin-fixed paraffin-embedded (FFPE) and cryostat-sliced sections.

\section{Materials And Methods}

\subsection{Photonic chip description and fabrication}

The photonic chip is composed of three layers: i) a bottom silicon (Si) substrate, ii) an intermediate cladding of silicon dioxide $\left(\mathrm{SiO}_{2}\right)$, and iii) a top waveguide layer of a high refractive index material made of either silicon nitride $\left(\mathrm{Si}_{3} \mathrm{~N}_{4}, n=2.0\right)$ or tantalum pentoxide $\left(\mathrm{Ta}_{2} \mathrm{O}_{5}, n=2.1\right)$ (see Fig. 1a). The high refractive index contrast (HIC) between the waveguide materials and the adjacent imaging medium and sample $(n \approx 1.4)$, allows the confinement and propagation of the excitation light via total internal reflection (TIR), enabling chip-based total internal reflection fluorescence microscopy (chip-TIRFM) (Fig. 1c). Diverse geometries have been previously studied for chip-TIRFM, including slab, rib, and strip waveguides [15]. Here, we chose uncladded strip waveguides with heights ranging from $150 \mathrm{~nm}$ to 250 $\mathrm{nm}$ and widths varying from $200 \mu \mathrm{m}$ to $1000 \mu \mathrm{m}$ (see Fig. 1b). 
In this study, we used both $\mathrm{Si}_{3} \mathrm{~N}_{4}$ and $\mathrm{Ta}_{2} \mathrm{O}_{5}$ chips for chip-TIRFM imaging of tissue sections. These were fabricated in distinct places: i) the $\mathrm{Si}_{3} \mathrm{~N}_{4}$ waveguide chips were manufactured according to CMOS fabrication process at the Institute of Microelectronics Barcelona (IMB-CNM, Barcelona, Spain) as detailed elsewhere $[15,17,58]$; ii) the $\mathrm{Ta}_{2} \mathrm{O}_{5}$ chips were manufactured at the Optoelectronics Research Center (ORC, University of Southampton, UK), following the process herewith detailed [59]. Waveguides of $250 \mathrm{~nm}$ thickness were fabricated by deposition of $\mathrm{Ta}_{2} \mathrm{O}_{5}$ film on a commercially-available 4" $\mathrm{Si}$ substrate having a $2.5 \mu \mathrm{m}$ thick $\mathrm{SiO}_{2}$ lower cladding layer (Si-Mat Silicon Materials, Germany) using a magnetron sputtering system (Plasmalab System 400, Oxford Instruments). The base pressure of the $\mathrm{Ta}_{2} \mathrm{O}_{5}$ deposition chamber was kept below $1 \times 10^{-6}$ Torr with Ar: $\mathrm{O}_{2}$ flow rates of $20 \mathrm{sccm}: 5 \mathrm{sccm}$ and the substrate temperature was maintained at $200^{\circ} \mathrm{C}$ throughout the deposition process. Photolithography was used to create a photoresist mask for further dry etching to fabricate strip waveguides. First, $1 \mu \mathrm{m}$ thick positive resist (Shipley, S1813) was coated on top of a $250 \mathrm{~nm} \mathrm{Ta}{ }_{2} \mathrm{O}_{5}$ film and then prebaked ( $1 \times$ $30 \mathrm{~min}$ ) at $90^{\circ} \mathrm{C}$. Then, the wafer was placed into a mask aligner (MA6, Süss MicroTec), and illuminated with the waveguide pattern. The $\mathrm{Ta}_{2} \mathrm{O}_{5}$ layer, which was not covered with photoresist, was fully etched to obtain strip waveguides of $250 \mathrm{~nm}$ height using an ion beam system (Ionfab 300+, Oxford Instruments) fed with argon at a flow rate of $6 \mathrm{SSCM}$. The process pressure $\left(2.3 \times 10^{-4} \mathrm{Torr}\right)$, beam voltage $(500 \mathrm{~V})$, beam current $(100 \mathrm{~mA})$, radiofrequency power $(500 \mathrm{~W})$, and substrate temperature $\left(15^{\circ} \mathrm{C}\right)$ were kept constant. Finally, the wafers were placed in a 3-zone semiconductor furnace at $600^{\circ} \mathrm{C}$ in an oxygen environment for 3 hours (in batch) to reduce the stress and supplement the oxygen deficiency created in $\mathrm{Ta}_{2} \mathrm{O}_{5}$ during the sputtering and the etching process [23].

Upon reception, the wafers were split into individual chips using a cleaving system (Latticegear, LatticeAx 225). The remaining photoresist layer from the manufacturing process was removed by immersion in acetone $(1 \times 1 \mathrm{~min})$. The chips were then cleaned in $1 \%$ Hellmanex in deionized water on a $70^{\circ} \mathrm{C}$ hotplate $(1 \times 10 \mathrm{~min})$, followed by rinsing steps with isopropanol and deionized water. The chips were finally dried with nitrogen using an air blowgun. To improve the adhesion of the tissue sections, the chips were rinsed with $0.1 \% \mathrm{w} / \mathrm{v}$ poly-L-lysine solution in $\mathrm{H}_{2} \mathrm{O}$ and let dry in a vertical position $(1 \times 30 \mathrm{~min})$.

\subsection{Sample collection and preparation}

\subsubsection{Ethical statement}

Both animal and human samples were handled according to relevant ethical guidelines. Healthy placental tissues were collected after delivery at the University Hospital of North Norway. Written consent was obtained from the participants following the protocol approved by the Regional Committee for Medical and Health Research Ethics of North Norway (REK Nord reference no. 2010/2058-4). Treatment and care of mice and pigs were conducted following the guidelines of the Norwegian Ethical and Welfare Board for Animal Research. Zebrafish experiments were conducted according to Swiss Laws and approved by the veterinary administration of the Canton of Zurich, Switzerland.

\section{Pranaration of Tnkuvacul cantinne for chip-based TIRFM, IFON, and SMLM} Loading [MathJax]/jax/output/CommonHTML/jax.js 
Human placental and murine (NZBxNZW)F1 kidney tissue samples were cryopreserved following the Tokuyasu method for ultracryotomy described elsewhere $[44,60]$. In short, biopsies blocks of approximately $1 \mathrm{~mm}^{3}$ were collected, rinsed in $9 \mathrm{mg} / \mathrm{mL}$ sodium chloride, fixed in $8 \%$ formaldehyde at $4^{\circ} \mathrm{C}$ overnight, infiltrated with $2.3 \mathrm{M}$ sucrose at $4^{\circ} \mathrm{C}$ overnight, mounted onto specimen pins, and frozen in liquid nitrogen. Thereafter, the samples were transferred to a cryo-ultramicrotome (EMUC6, Leica Microsystems) and sectioned with a diamond knife into thin slices ranging from $100 \mathrm{~nm}$ to $1 \mu \mathrm{m}$ thickness. The sections were collected with a wire loop containing a 1:1 cryoprotectant mixture of $2 \%$ methylcellulose and 2.3 M sucrose and transferred to photonic chips coated with poly-L-lysine and equipped with custom-made polydimethylsiloxane (PDMS) chambers of approximately $130 \mu \mathrm{m}$-height [17] (Figure 1b). The samples were stored on Petri dishes at $4^{\circ} \mathrm{C}$ before subsequent steps.

Diverse staining strategies were employed according to each imaging modality:

i) For Chip-based multicolor TIRFM imaging, human placental sections of $400 \mathrm{~nm}$ were direct-labeled for membranes, F-actin, and nuclei as described herewith. First, the cryoprotectant mixture was dissolved by incubating the samples in phosphate-buffered saline (PBS) $(3 \times 10 \mathrm{~min})$ at $37^{\circ} \mathrm{C}$. Thereafter, the samples were incubated in a 1:2000 solution of CellMask Deep Red in PBS $(1 \times 15 \mathrm{~min})$ at room temperature (RT) and subsequently washed with PBS $(2 \times 5 \mathrm{~min})$. Next, the sections were incubated in 1:100 PhalloidinAtto565 in PBS $(1 \times 15 \mathrm{~min})$ and washed with PBS $(2 \times 5 \mathrm{~min})$. Further, the samples were incubated in 1:500 Sytox Green in PBS $(1 \times 10 \mathrm{~min})$ and washed with PBS $(2 \times 5 \mathrm{~min})$. Finally, the sections were mounted with \#1.5 coverslips using Prolong Diamond and sealed with Picodent Twinsil.

ii) For Chip-based SMLM imaging, mouse kidney cryosections of $400 \mathrm{~nm}$ were labeled for membranes and nuclei using CellMask Deep Red and Sytox Green, respectively, following identical concentrations and incubation steps as for the Chip-based multicolor TIRFM imaging To enable photoswitching of the fluorescent molecules, the samples were mounted with a water-based enzymatic oxygen scavenging system buffer as described in previous works $[15,16]$. Thereafter, the sections were covered with \#1.5 coverslips and sealed with Picodent Twinsil.

iii) For Chip-based IFON imaging, human placental sections of $400 \mathrm{~nm}$ were prepared identically to the Chip-based multicolor TIRFM imaging experiment, except for the membrane labeling and subsequent washing steps that were omitted. In all cases, the labeled cryosections were stored at $4^{\circ} \mathrm{C}$ and protected from light before imaging. Supplementary Information $S 8$ provides a detailed description of the materials and reagents used in this protocol.

\subsubsection{Preparation of Tokuyasu sections for chip-based CLEM}

For Chip-based CLEM imaging, zebrafish eyes were prepared as described elsewhere [61]. Briefly, 5 dayspost-fertilization larvae were euthanized in tricaine and fixed with $4 \%$ formaldehyde and $0.025 \%$ glutaraldehyde in $0.1 \mathrm{M}$ sodium cacodylate buffer $(1 \times 16 \mathrm{~h})$ at $4{ }^{\circ} \mathrm{C}$. Subsequently, eyes were dissected and washed in PBS, placed in $12 \%$ gelatin $(1 \times 10 \mathrm{~min})$ at $40^{\circ} \mathrm{C}$, and finally left to harden at $4^{\circ} \mathrm{C}$.

Loading [MathJax]/jax/output/CommonHTML/jax.js ;rose and stored at $4^{\circ} \mathrm{C}$ before further storage in liquid

Page 15/28 
nitrogen. Ultrathin sections of $110 \mathrm{~nm}$ thickness were obtained with a cryo-ultramicrotome (Ultracut EM FC6, Leica Microsystems) using a cryo-immuno diamond knife ( $35^{\circ}$ - size $2 \mathrm{~mm}$, Diatome). The cryosections were transferred to photonic chips fitted with a PDMS frame and stored at $4{ }^{\circ} \mathrm{C}$ before staining. The samples were incubated in PBS $(1 \times 20 \mathrm{~min})$ at $0{ }^{\circ} \mathrm{C}$, followed by two washing steps in PBS $(2 \times 2 \mathrm{~min})$ at RT to dissolve the cryoprotectant. Then, the samples were pre-incubated with a blocking solution (PBG) for $5 \mathrm{~min}$, followed by incubation $(1 \times 45 \mathrm{~min})$ in a 1:50 solution of rabbit anti-Tomm20 in PBG blocking buffer at RT. After several rinsing $(6 \times 2 \mathrm{sec})$ and washing $(1 \times 5 \mathrm{~min})$ in PBG, the specimens were incubated $(1 \times 45 \mathrm{~min})$ with an Alexa Fluor 647-conjugated secondary donkey anti-rabbit antibody at 1:200 concentration in PBG at RT. For the acting staining, the samples were washed in PBS (6 $\times 1 \mathrm{~min})$, followed by incubation with Texas Red-X Phalloidin $(1 \times 10 \mathrm{~min})$ at 1:50 concentration in PBS. After washes in PBS ( $2 \times 5 \mathrm{~min})$, the samples were incubated in a 1:500 solution of Sytox Green nuclear staining in PBS $(1 \times 10 \mathrm{~min})$, followed by washes in PBS $(2 \times 5 \mathrm{~min})$, and mounting with a 1:1 mixture of PBS and glycerol (49782, Sigma-Aldrich) and covered with a \#1.5 glass coverslip before chip-TIRFM imaging. Supplementary Information S8 provides a detailed description of the materials and reagents used in this protocol

\subsection{Chip imaging and processing}

\subsubsection{Chip-based imaging}

The chip-TIRFM setup was assembled using a modular upright microscope (BXFM, Olympus), together with a custom-built photonic chip module as shown in Figure 1c and Supplementary Information S11. A fiber-coupled multi-wavelength laser light source (iChrome CLE, Toptica) was expanded and collimated through an optical fiber collimator (F280APC-A, Thorlabs) to fill the back aperture of the coupling MO (NPlan 50X/NA0.5, Olympus). Typical illumination wavelengths used were $\mathrm{I}_{1}=640 \mathrm{~nm}, \mathrm{I}_{2}=561 \mathrm{~nm}$, and $\mathrm{I}_{3}=488 \mathrm{~nm}$. Both the optical fiber collimator and the coupling objective were mounted on an XYZ translation stage (Nanomax300, Thorlabs) fitted with an XY piezo-controllable platform (Q-522 Q-motion, $\mathrm{PI})$ for fine adjustments of the coupling light into the waveguides. The photonic chips were placed on a custom-made vacuum chuck fitted on an X-axis translation stage (XRN25P, Thorlabs) for large-range scanning of parallel waveguides. Fluorescent emission of the samples was achieved via evanescent field excitation upon coupling of the laser onto a chosen waveguide, as detailed elsewhere [15] (Figure 1a,c). Various $\mathrm{MO}$ lenses were used to collect the fluorescent signal, depending on the desired FOV, magnification, and resolution (4X/0.1NA, 20X/0.45NA, and 60X/1.2NA water immersion). An emission filter set composed of a long-pass filter and a band-pass filter was used to block out the excitation signal at each wavelength channel (see Supplementary Information S11 for details). The emission signal passed through the microscope's $1 \mathrm{X}$ tube lens (U-TV1X-2, Olympus) before reaching the sCMOS camera image plane (Orca-flash4.0, Hamamatsu). Both the camera exposure time and the laser intensity were adjusted according to the experimental goal. For TIRFM imaging, the camera exposure time was set between $50 \mathrm{~ms}$ and $100 \mathrm{~ms}$, and the input power was incrementally adjusted until the mean histogram values surpassed 500 counts. For SMLM, the acquisition time was set to $30 \mathrm{~ms}$ while the input power 
input powers were between $10 \%$ and $60 \%$ for TIRFM imaging, and between $90 \%$ to $100 \%$ for SMLM imaging. To reduce photobleaching of the fluorescent markers, the image acquisition was sequentially performed from less energetic to more energetic excitation wavelengths. To deal with the anisotropic mode distribution of the multi-mode interference pattern at the waveguide, the coupling objective was laterally scanned at $<1 \mathrm{~mm}$ steps over a $50 \mu \mathrm{m}-200 \mu \mathrm{m}$ travel span along the input facet of the chip while individual images were taken. Image stacks of various sizes were acquired according to the imaging technique. Typically, $100-1000$ frames for TIRFM and $30000-50000$ frames for SMLM. White light from a halogen lamp (KL1600 LED, Olympus) was used for bright-field illumination to identify the regions of interest $(\mathrm{ROI})$ through the collection objective. To reduce mechanical instability, the collection path of the system was fixed to the optical table, while the photonic chip module was placed onto a motorized stage (8MTF, Standa) for scanning across the XY directions. An optical table (CleanTop, TMC) was used as the main platform for the chip-TIRFM setup. Supplementary Information S11 offers a detailed description of the chip-TIRFM setup.

\subsubsection{CLEM imaging}

After chip-TIRFM imaging, both the coverslip and the PDMS frame were removed and the samples fixed with $0.1 \%$ glutaraldehyde. Thereafter, the samples were incubated with methylcellulose followed by centrifugation at $4700 \mathrm{rpm}$ (Heraeus Megafuge 40R, Thermo Scientific) in a falcon tube. After drying ( $2 \times$ $10 \mathrm{~min}$ ) at $40{ }^{\circ} \mathrm{C}$ on a heating plate, the photonic chips were transferred to an electron beam evaporator (MED 020, Leica Microsystems). The specimen was then coated with platinum/carbon (Pt/C, $10 \mathrm{~nm})$ by rotary shadowing at an angle of 8 degrees [48]. Thereafter, the photonic chips were mounted on a $25 \mathrm{~mm}$ Pin Mount SEMclip (\#16144-9-30, Ted Pella) and imaged at $4 \mathrm{~nm}$ pixel size with a scanning electron microscope (Auriga 40 CrossBeam, Carl Zeiss Microscopy) at a low-accelerating voltage (1.5 keV). Supplementary Information S12 illustrates various steps of SEM imaging on a photonic chip.

\subsubsection{Image processing}

The acquired frames were computationally processed on the open-source software Fiji [62] according to the desired imaging technique. To obtain diffraction-limited TIRFM images, the image stacks were computationally averaged using the $Z$ Project tool. Thereafter, the averaged images were deconvolved with the DeconvolutionLab2 plugin [63], using a synthetic 2D point spread function (PSF) matching the effective pixel size of the optical system. Lastly, the Merge Channels tool was used to merge and pseudocolor independent averaged channels into a multicolor composite TIRFM image. SMLM images were reconstructed using the thunderSTORM plugin [64]. For CLEM, the acquired TIRFM stacks were first processed with the NanoJ SRRF plugin [65] and then correlated with the EM images using the TrakEM2 plugin [66].

\section{Declarations}

\section{Acknowledgments and funding}


The authors thank the collaborators at UiT The Arctic University of Norway, including Randi Olsen for providing the cryosections, and Deanna Wolfson for her valuable labeling recommendations. The authors also acknowledge Åsa Birna Birgisdottir and Trine Kalstad, for providing the pig heart samples, and Prof. Dr. Stephan Neuhauss, University of Zurich, for providing the zebrafish eye samples. The authors would like to express their appreciation to Prof. James Wilkinson (University of Southampton) and Dr. Senthil Murugan Ganapathy (University of Southampton) for discussions on the waveguide platform fabrication. B.S.A. acknowledges the funding from the Research Council of Norway, project \# NANO 2021-288565 and project \# BIOTEK 2021-285571.

\section{Author contribution}

B.S.A. conceived the idea and supervised the project. L.E.V.H. and V.D. planned and coordinated the experiments, performed sample labeling, chip-TIRFM imaging, and post-processing of the data. J.C.T. and V.D. built the chip-based microscope setup. S.A. and K.A. performed MUSICAL reconstruction. D.C., V.D., and L.E.V.H. performed SMLM acquisition. D.C. performed the dSTORM reconstruction. L.E.V.H., J.C.T., and J.M.M. performed chip-TIRFM imaging of the zebrafish eye. G.B. provided the zebrafish cryosections. G.B, J.M.M., and U.Z. designed the experimental conditions for SEM imaging. J.M.M. performed the SEM imaging for CLEM. F.T.D. and A.P. respectively designed and fabricated the $\mathrm{Ta}_{2} \mathrm{O}_{5}$ photonic chips. G.A. provided the human placental sample. M.N. collected and preserved the human placental samples. G.A. and M.N. helped with the placental image interpretations. A.K.H. and K.A.F. collected and preserved the mouse kidney tissue and assisted with the renal image interpretations. L.E.V.H. and V.D. analyzed the data, prepared the figures. L.E.V.H., V.D., and B.S.A. wrote the manuscript. All authors contributed to writing and revising selected sections of the manuscript.

\section{Conflicts of interest}

B.S.A. has applied for a patent for chip-based optical nanoscopy and he is co-founder of the company Chip Nanolmaging AS, which commercializes on-chip super-resolution microscopy systems.

\section{References}

1. Schlichenmeyer, T.C., et al., Video-rate structured illumination microscopy for high-throughput imaging of large tissue areas. Biomedical optics express, 2014. 5(2): p. 366-377.

2. Aeffner, F., et al., Digital microscopy, image analysis, and virtual slide repository. ILAR journal, 2018. 59(1): p. 66-79.

3. Pullman, J.M., New views of the glomerulus: advanced microscopy for advanced diagnosis. Frontiers in Medicine, 2019. 6: p. 37.

4. Tosoni, A., G. Barbiano di Belgiojoso, and M. Nebuloni, Electron microscopy in the diagnosis of amyloidosis. Amyloidosis-mechanisms and prospects for therapy, 2011: p. 121-148.

5. Hell, S.W., et al., The 2015 super-resolution microscopy roadmap. Journal of Physics D: Applied 
6. Schermelleh, L., R. Heintzmann, and H. Leonhardt, $A$ guide to super-resolution fluorescence microscopy. Journal of Cell Biology, 2010. 190(2): p. 165-175.

7. Sahl, S.J., S.W. Hell, and S. Jakobs, Fluorescence nanoscopy in cell biology. Nature reviews Molecular cell biology, 2017. 18(11): p. 685.

8. Booth, M., et al., Aberrations and adaptive optics in super-resolution microscopy. Microscopy, 2015. 64(4): p. 251-261.

9. Baschong, W., R. Suetterlin, and R.H. Laeng, Control of autofluorescence of archival formaldehydefixed, paraffin-embedded tissue in confocal laser scanning microscopy (CLSM). Journal of Histochemistry \& Cytochemistry, 2001. 49(12): p. 1565-1571.

10. Creech, M.K., et al., Superresolution imaging of clinical formalin fixed paraffin embedded breast cancer with single molecule localization microscopy. Scientific reports, 2017. 7(1): p. 1-10.

11. Wang, M., et al., High-resolution rapid diagnostic imaging of whole prostate biopsies using video-rate fluorescence structured illumination microscopy. Cancer research, 2015. 75(19): p. 4032-4041.

12. Ilgen, P., et al., STED super-resolution microscopy of clinical paraffin-embedded human rectal cancer tissue. PloS one, 2014. 9(7): p. e101563.

13. Göttfert, F., et al., Coaligned dual-channel STED nanoscopy and molecular diffusion analysis at 20 $n m$ resolution. Biophysical journal, 2013. 105(1): p. L01-L03.

14. Agarwal, K. and R. Macháň, Multiple signal classification algorithm for super-resolution fluorescence microscopy. Nature communications, 2016. 7(1): p. 1-9.

15. Diekmann, R., et al., Chip-based wide field-of-view nanoscopy. Nature Photonics, 2017. 11(5): p. 322.

16. Coucheron, D.A., et al. Chip-based nanoscopy: towards integration and high-throughput imaging. in Nanoimaging and Nanospectroscopy V. 2017. International Society for Optics and Photonics.

17. Tinguely, J.-C., Ø.I. Helle, and B.S. Ahluwalia, Silicon nitride waveguide platform for fluorescence microscopy of living cells. Optics express, 2017. 25(22): p. 27678-27690.

18. Opstad, I.S., et al., A waveguide imaging platform for live-cell TIRF imaging of neurons over large fields of view. Journal of Biophotonics, 2020. 13(6): p. e201960222.

19. Coucheron, D.A., et al., High-Throughput Total Internal Reflection Fluorescence and Direct Stochastic Optical Reconstruction Microscopy Using a Photonic Chip. JoVE (Journal of Visualized Experiments), 2019(153): p. e60378.

20. Helle, Ø.І., et al., Nanoscopy on-a-chip: super-resolution imaging on the millimeter scale. Optics express, 2019. 27(5): p. 6700-6710.

21. Agnarsson, B., et al., On-chip modulation of evanescent illumination and live-cell imaging with polymer waveguides. Optics express, 2011. 19(23): p. 22929-22935.

22. Griffiths, G., J.-W. Slot, and P. Webster, Kiyoteru Tokuyasu: a pioneer of cryo-ultramicrotomy. 2015, The Japanese Society of Microscopy.

23. Priyadarshi, A., et al., A transparent waveguide chip for versatile TIRF-based microscopy and 
24. Dubey, V., et al., Multi-modal chip-based fluorescence and quantitative phase microscopy for studying inflammation in macrophages. Optics express, 2018. 26(16): p. 19864-19876.

25. Mittler, S., Waveguide Evanescent Field Fluorescence and Scattering Microscopy: The Status Quo, in Optics, Photonics and Laser Technology. 2018, Springer. p. 1-24.

26. Jayakumar, N., et al., On-chip TIRF nanoscopy by applying Haar wavelet kernel analysis on intensity fluctuations induced by chip illumination. Optics Express, 2020. 28(24): p. 35454-35468.

27. Archetti, A., et al., Waveguide-PAINT offers an open platform for large field-of-view super-resolution imaging. Nature communications, 2019. 10(1): p. 1-9.

28. Helle, Ø.I., et al., Structured illumination microscopy using a photonic chip. Nature Photonics, 2020: p. 1-8.

29. Tinguely, J.-C., et al., Photonic-chip assisted correlative light and electron microscopy. Communications biology, 2020. 3(1): p. 1-7.

30. Silini, A.R., et al., Perinatal derivatives: where do we stand? A roadmap of the human placenta and consensus for tissue and cell nomenclature. Frontiers in Bioengineering and Biotechnology, 2020. 8 : p. 1438.

31. Tokuyasu, K., A technique for ultracryotomy of cell suspensions and tissues. The Journal of cell biology, 1973. 57(2): p. 551-565.

32. Tokuyasu, K., A study of positive staining of ultrathin frozen sections. Journal of ultrastructure research, 1978. 63(3): p. 287-307.

33. Loussert-Fonta, C., et al., Correlation of fluorescence microscopy, electron microscopy, and NanoSIMS stable isotope imaging on a single tissue section. Communications biology, 2020. 3(1): p. 1-9.

34. Kopek, B.G., et al., Diverse protocols for correlative super-resolution fluorescence imaging and electron microscopy of chemically fixed samples. Nature protocols, 2017. 12(5): p. 916-946.

35. Askelund, K. and L. Chamley, Trophoblast deportation part I: review of the evidence demonstrating trophoblast shedding and deportation during human pregnancy. Placenta, 2011. 32(10): p. 716-723.

36. King, B.F., The organization of actin filaments in human placental villi. Journal of ultrastructure research, 1983. 85(3): p. 320-328.

37. Van de Linde, S., et al., Direct stochastic optical reconstruction microscopy with standard fluorescent probes. Nature protocols, 2011. 6(7): p. 991.

38. Carlson, E.C., et al., Significant glomerular basement membrane thickening in hyperglycemic and normoglycemic diabetic-prone BB Wistar rats. The Anatomical Record Part A: Discoveries in Molecular, Cellular, and Evolutionary Biology: An Official Publication of the American Association of Anatomists, 2004. 281(2): p. 1308-1318.

39. Opstad, I.S., et al., Fluorescence fluctuations-based super-resolution microscopy techniques: an experimental comparative study. arXiv preprint arXiv:2008.09195, 2020.

40. Miura, S., et al., Fluid shear triggers microvilli formation via mechanosensitive activation of TRPV6. Nature nommuniratione $2015 \mathrm{~h}(1) \cdot \mathrm{n}$ 1-11.

Loading [MathJax]/jax/output/CommonHTML/jax.js

Page 20/28 
41. Redman, C., et al., Does size matter? Placental debris and the pathophysiology of pre-eclampsia. Placenta, 2012. 33: p. S48-S54.

42. Lange, K., Fundamental role of microvilli in the main functions of differentiated cells: Outline of an universal regulating and signaling system at the cell periphery. Journal of cellular physiology, 2011. 226(4): p. 896-927.

43. Acuña, S., et al., Soft thresholding schemes for multiple signal classification algorithm. Optics Express, 2020. 28(23): p. 34434-34449.

44. Villegas-Hernández, L.E., et al., Visualizing ultrastructural details of placental tissue with superresolution structured illumination microscopy. Placenta, 2020. 97: p. 42-45.

45. Wang, M., et al., Gigapixel surface imaging of radical prostatectomy specimens for comprehensive detection of cancer-positive surgical margins using structured illumination microscopy. Scientific reports, 2016. 6(1): p. 1-16.

46. Johnson, K.A. and G.M. Hagen, Artifact-free whole-slide imaging with structured illumination microscopy and Bayesian image reconstruction. GigaScience, 2020. 9(4): p. giaa035.

47. Mateos, J.M., et al., Correlative super-resolution and electron microscopy to resolve protein localization in zebrafish retina. Journal of visualized experiments: JoVE, 2017(129).

48. Mateos, J.M., et al., Topographic contrast of ultrathin cryo-sections for correlative super-resolution light and electron microscopy. Scientific reports, 2016. 6(1): p. 1-8.

49. Link, B.A. and R.F. Collery, Zebrafish models of retinal disease. Annual review of vision science, 2015. 1: p. 125-153.

50. Reichelt, M., et al., 3D reconstruction of VZV infected cell nuclei and PML nuclear cages by serial section array scanning electron microscopy and electron tomography. PLoS Pathog, 2012. 8(6): p. e1002740.

51. Arganda-Carreras, I., et al., 3D reconstruction of histological sections: application to mammary gland tissue. Microscopy research and technique, 2010. 73(11): p. 1019-1029.

52. Werbin, J.L., et al., Multiplexed Exchange-PAINT imaging reveals ligand-dependent EGFR and Met interactions in the plasma membrane. Scientific reports, 2017. 7(1): p. 1-12.

53. Løvhaugen, P., et al., Serial Raman spectroscopy of particles trapped on a waveguide. Optics express, 2013. 21(3): p. 2964-2970.

54. Helle, Ø.I., B.S. Ahluwalia, and O.G. Hellesø, Optical transport, lifting and trapping of micro-particles by planar waveguides. Optics express, 2015. 23(5): p. 6601-6612.

55. Wang, X., et al., Enhanced cell sorting and manipulation with combined optical tweezer and microfluidic chip technologies. Lab on a Chip, 2011. 11(21): p. 3656-3662.

56. Orth, A. and K. Crozier, Gigapixel fluorescence microscopy with a water immersion microlens array. Optics express, 2013. 21(2): p. 2361-2368.

57. Cappi, G., et al., Ultra-fast and automated immunohistofluorescent multistaining using a microfluidic ticclle nrenecenr Srientifir rennrts 2n19. 9(1): p. 1-12.

Loading [MathJax]/jax/output/CommonHTML/jax.js 
58. Coucheron, D.A., et al. Chip-based nanoscopy: towards integration and high-throughput imaging. in SPIE Nanoscience + Engineering. 2017. SPIE.

59. Ahluwalia, B.S., et al., Fabrication of submicrometer high refractive index Tantalum Pentoxide waveguides for optical propulsion of microparticles. IEEE Photonics Technology Letters, 2009. 21(19): p. 1408-1410.

60. Fenton, K., et al., Anti-dsDNA antibodies promote initiation, and acquired loss of renal Dnase1 promotes progression of lupus nephritis in autoimmune (NZBXNZW) F1 mice. PloS one, 2009. 4(12): p. e8474.

61. Mateos, J.M., et al., Correlative super-resolution and electron microscopy to resolve protein localization in zebrafish retina. JoVE (Journal of Visualized Experiments), 2017(129): p. e56113.

62. Schindelin, J., et al., Fiji: an open-source platform for biological-image analysis. Nat Meth, 2012. 9(7): p. 676-682.

63. Sage, D., et al., Deconvolution Lab2: An open-source software for deconvolution microscopy. Methods, 2017. 115: p. 28-41.

64. Ovesný, M., et al., ThunderSTORM: a comprehensive ImageJ plug-in for PALM and STORM data analysis and super-resolution imaging. Bioinformatics, 2014. 30(16): p. 2389-2390.

65. Gustafsson, N., et al., Fast live-cell conventional fluorophore nanoscopy with ImageJ through superresolution radial fluctuations. Nature Communications, 2016. 7(1): p. 12471.

66. Cardona, A., et al., TrakEM2 software for neural circuit reconstruction. PloS one, 2012. 7(6): p. e38011.

\section{Figures}




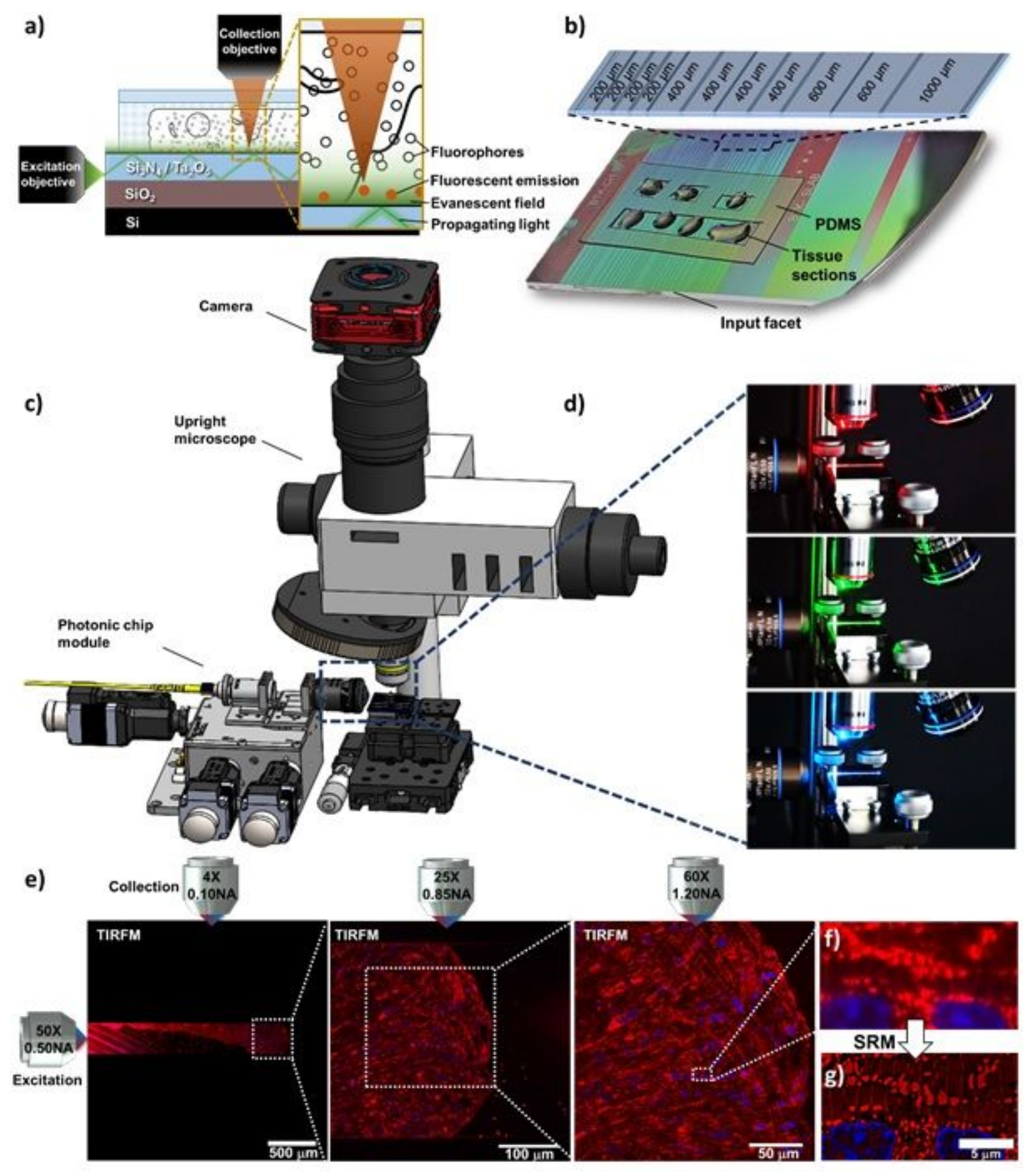

Figure 1

Schematic representation of the chip-based total internal reflection fluorescence microscopy (chipTIRFM) setup. (a) Working principle of chip-TIRFM: upon coupling onto the input facet, the excitation light propagates through the waveguide core material due to total internal reflection. An evanescent field of approx. $150 \mathrm{~nm}$ height excites a thin layer of fluorescent dyes in the vicinity of the photonic chip surface, allowing for TIRFM imaging. (b) Top view of a photonic chip containing ultrathin Tokuyasu cryosections covered with a 1:1 cryoprotectant mixture of $2.3 \mathrm{M}$ sucrose and $2 \%$ methylcellulose, and surrounded by a custom-made transparent polydimethylsiloxane (PDMS) frame. The inset illustrates the various strip unvanuidn widthe nunilahln $n n$ thn nhin In) The chip-TIRFM setup is composed of a custom-made Loading [MathJax]/jax/output/CommonHTML/jax.js 
photonic chip module and a commercially available upright collection module. Upon coupling the excitation light on the photonic chip, the fluorescent signal is allowed through a filter set and captured with a scientific CMOS camera. (d) The photonic chip allows decoupling of the excitation and the collection light paths, enabling TIRFM imaging using conventional microscope objectives. Different wavelengths propagating on the waveguide core allow for multicolor TIRFM imaging. (e) TIRFM images of a $100 \mathrm{~nm}$ thick pig heart cryosection imaged on a photonic chip through different microscope objectives. Membranes in red and nuclei in blue. (f) Magnified view of the diffraction-limited TIRFM image acquired with a 60X/1.20NA water immersion microscope objective. (g) Subsequent postprocessing of the raw data enables super-resolution microscopy (SRM), allowing the visualization of structures beyond the diffraction limit of conventional optical microscopy.

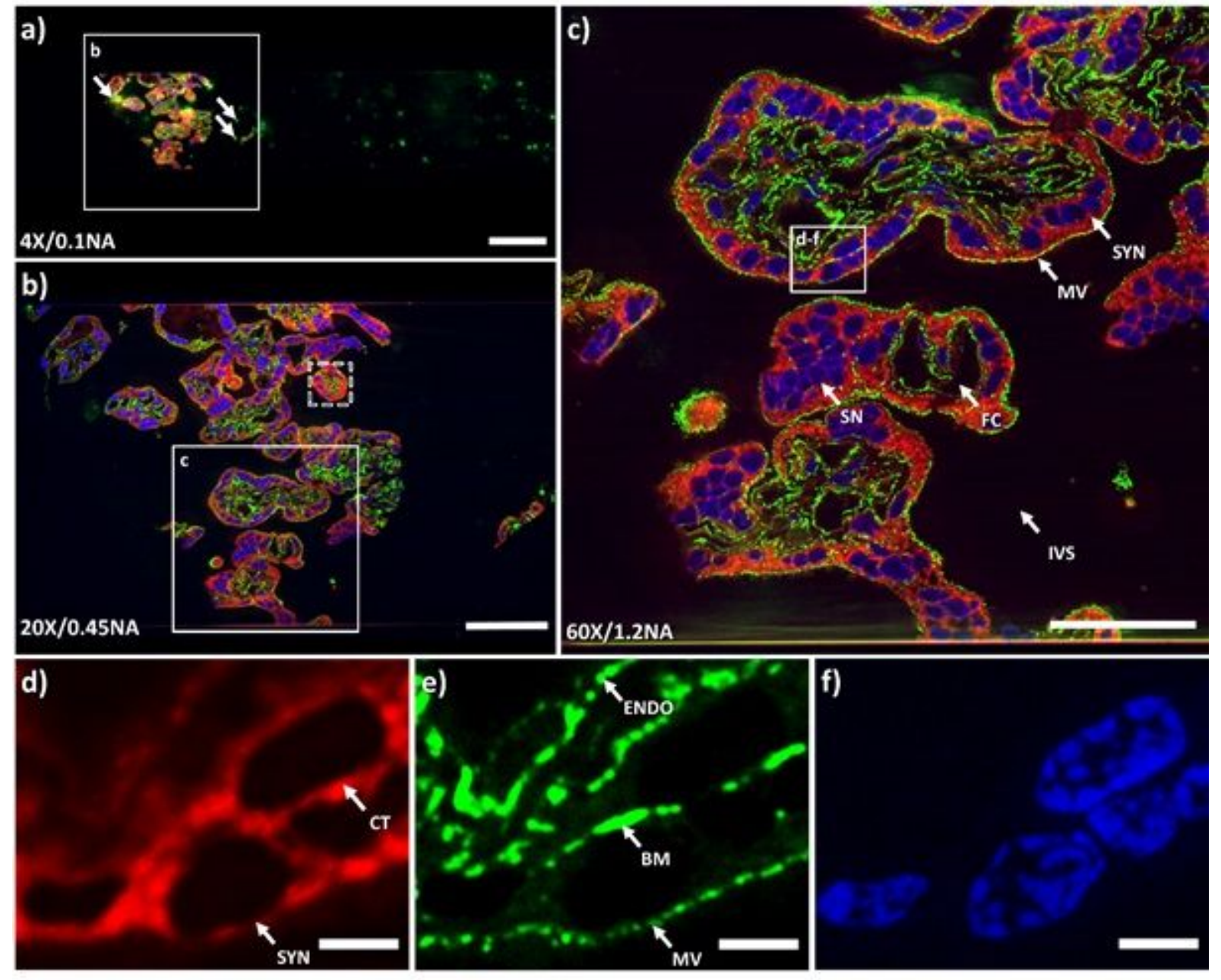

Figure 2

Chip-based multicolor TIRFM imaging of a $400 \mathrm{~nm}$ placental tissue section prepared by Tokuyasu method. Membranes labeled with CellMask Deep Red (pseudo-colored in red), F-actin labeled with Phalloidin-Atto565 (pseudo-colored in green), and nuclei labeled with Sytox Green (pseudo-colored in blue). (a) Large field of view chip-based multicolor TIRFM image acquired with a 4X/0.1NA microscope objective. The white arrows indicate the locations of unspecific binding of the F-actin marker to the wavequide. The white box represents the area imaged with a higher magnification objective lens in (b). Loading [MathJax]/jax/output/CommonHTML/jax.js 
(b) Chip-based multicolor TIRFM image acquired with a 20X/0.45NA microscope objective. The white box represents the area subsequently imaged with a higher magnification objective lens in (c). The whitedotted box illustrates the maximum field of view $(50 \mu \mathrm{m} \times 50 \mu \mathrm{m})$ attainable in a conventional TIRFM setup. (c) Multicolor chip-TIRFM image acquired with a 60X/1.2NA microscope objective allows the identification of morphologically relevant structures of the chorionic villi such as the syncytiotrophoblastic cells (SYN), fetal capillaries (FC), syncytial knots (SN), and intervillous space (IVS) without maternal red blood cells due to thorough rinsing during sample preparation. The white box corresponds to the individual channels magnified in (d-f). (d) A magnified view of the membrane signal allows the distinction between a SYN and a cytotrophoblastic cell (CT). (e) A magnified view of the Factin signal conforms to the expected location for this marker, in places such as the microvilli brush border (MV), the SYN's basal membrane (BM), and the capillary endothelial cell (ENDO). (f) Magnified view of syncytial and cytotrophoblast nuclei. Scale bars (a) $200 \mu \mathrm{m}$, (b) $100 \mu \mathrm{m}$, (c) $50 \mu \mathrm{m}$, (d-e) $5 \mu \mathrm{m}$.
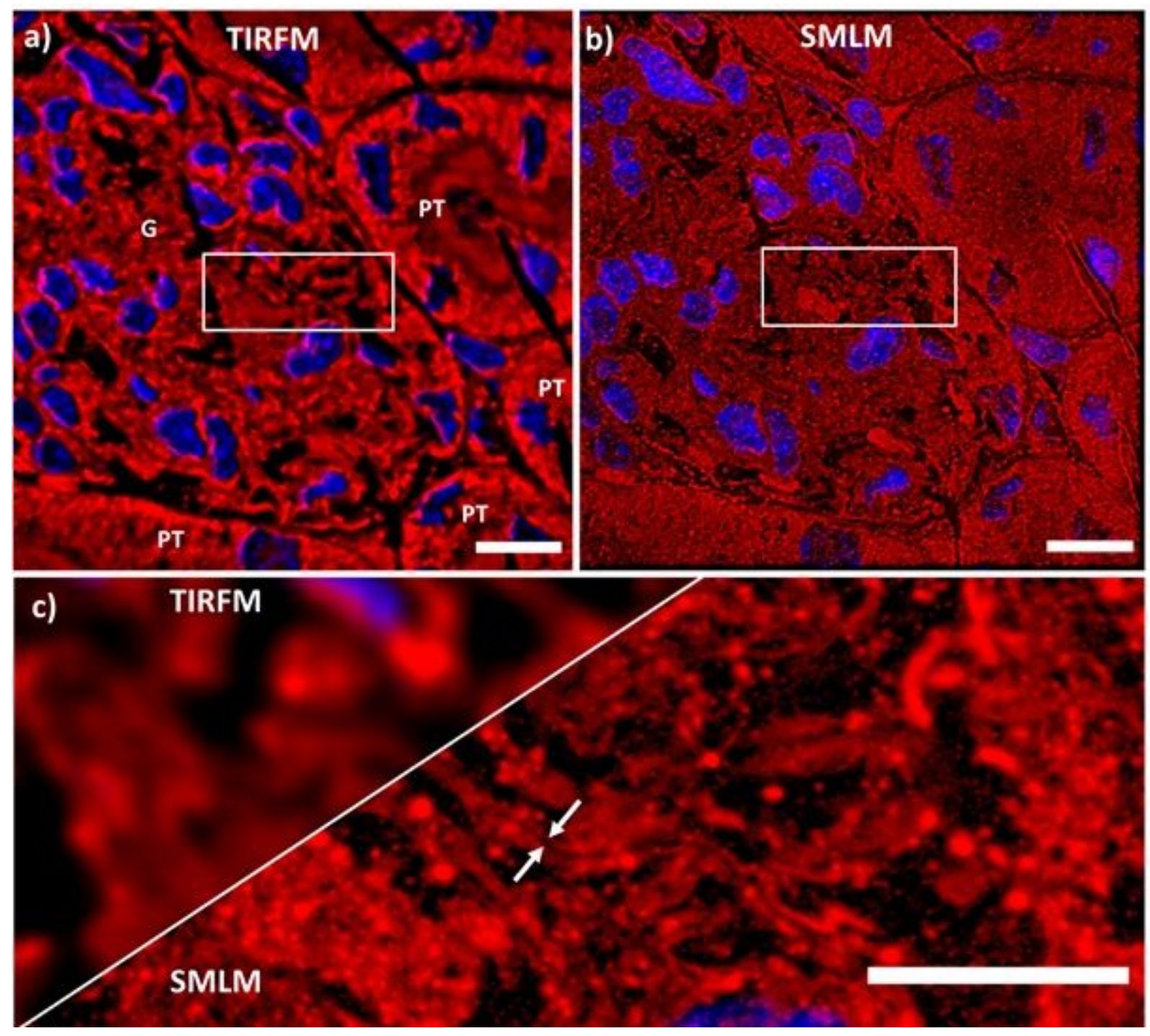

Figure 3

Chip-based single-molecule localization microscopy of a $400 \mathrm{~nm}$ mouse kidney cryosections prepared by Tokuyasu method. Membranes labeled with CellMask Deep Red (pseudo-colored in red), and nuclei Loading [MathJax]/jax/output/CommonHTML/jax.js 
labeled with Sytox Green (pseudo-colored in blue). Images were collected using a 60X/1.2 NA water immersion microscope objective. (a) Chip-TIRFM image of a glomerulus (G) surrounded by proximal tubuli (PT). (b) Chip-based SMLM image reconstructed with dSTORM algorithm. (c) A magnified view of the white rectangles in (a-b) allows a comparison between these two imaging techniques. In particular, the white arrows in the SMLM segment show two lines with an average separation of $\sim 100 \mathrm{~nm}$ that is otherwise not observable in the TIRFM segment. Arguably, this ultrastructural feature is in agreement with the dimensions of the glomerular basal membrane present in this filtration compartment of the kidney. Scale bars (a-b) $10 \mu \mathrm{m}$, (c) $5 \mu \mathrm{m}$.
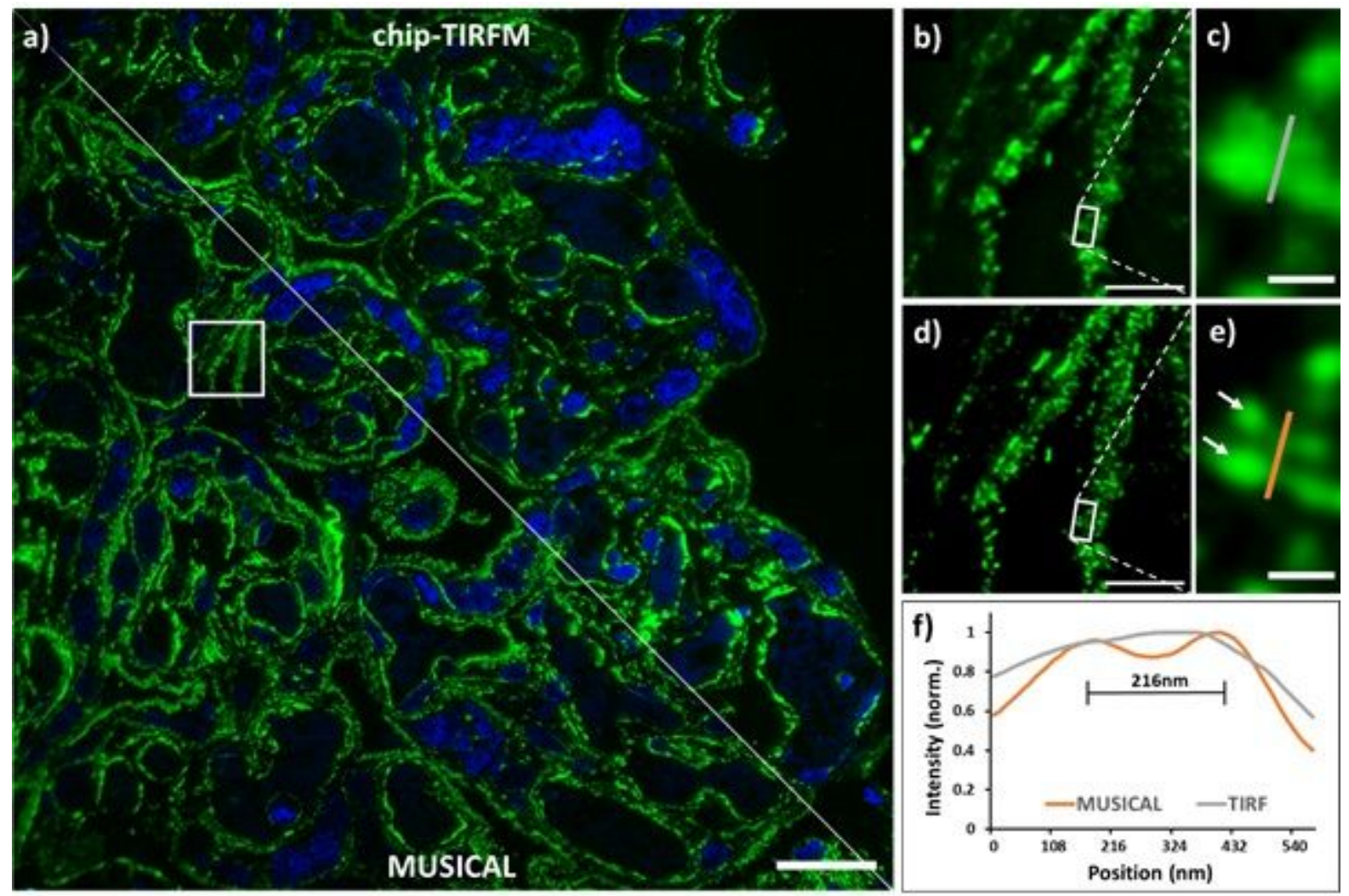

\section{Figure 4}

Chip-based intensity fluctuation optical nanoscopy of a $400 \mathrm{~nm}$ thick placental tissue section prepared per Tokuyasu method. F-actin labeled with Phalloidin-Atto565 (pseudo-colored in green), and nuclei labeled with Sytox Green (pseudo-colored in blue). (a) Multicolor fluorescent image over a 220 x $220 \mu \mathrm{m} 2$ FOV acquired with a 60x/1.2NA microscope objective. A solid white line divides the image into two segments, illustrating the averaged chip-TIRFM on the top and the MUSICAL reconstruction at the bottom. $(b, d)$ A magnified view of the white box in (a) allows for visualization of the microvilli (MV) lining the syncytiotrophoblast's brush border. (c) Further magnification of the white box in (b) shows a single structure. (e) White arrows denote the location of two adjacent MV over the magnified white box in (d). (f) Line-profile measurements reveal a separation of $216 \mathrm{~nm}$ between two adjacent MV on the MUSICAL reconstruction in (e) that is otherwise not distinguishable on the averaged chip-TIRFM image in (c). Scale bars (a) $20 \mathrm{~mm},(b, d) 5 \mathrm{~mm}$, and (c,e) $500 \mathrm{~nm}$. 

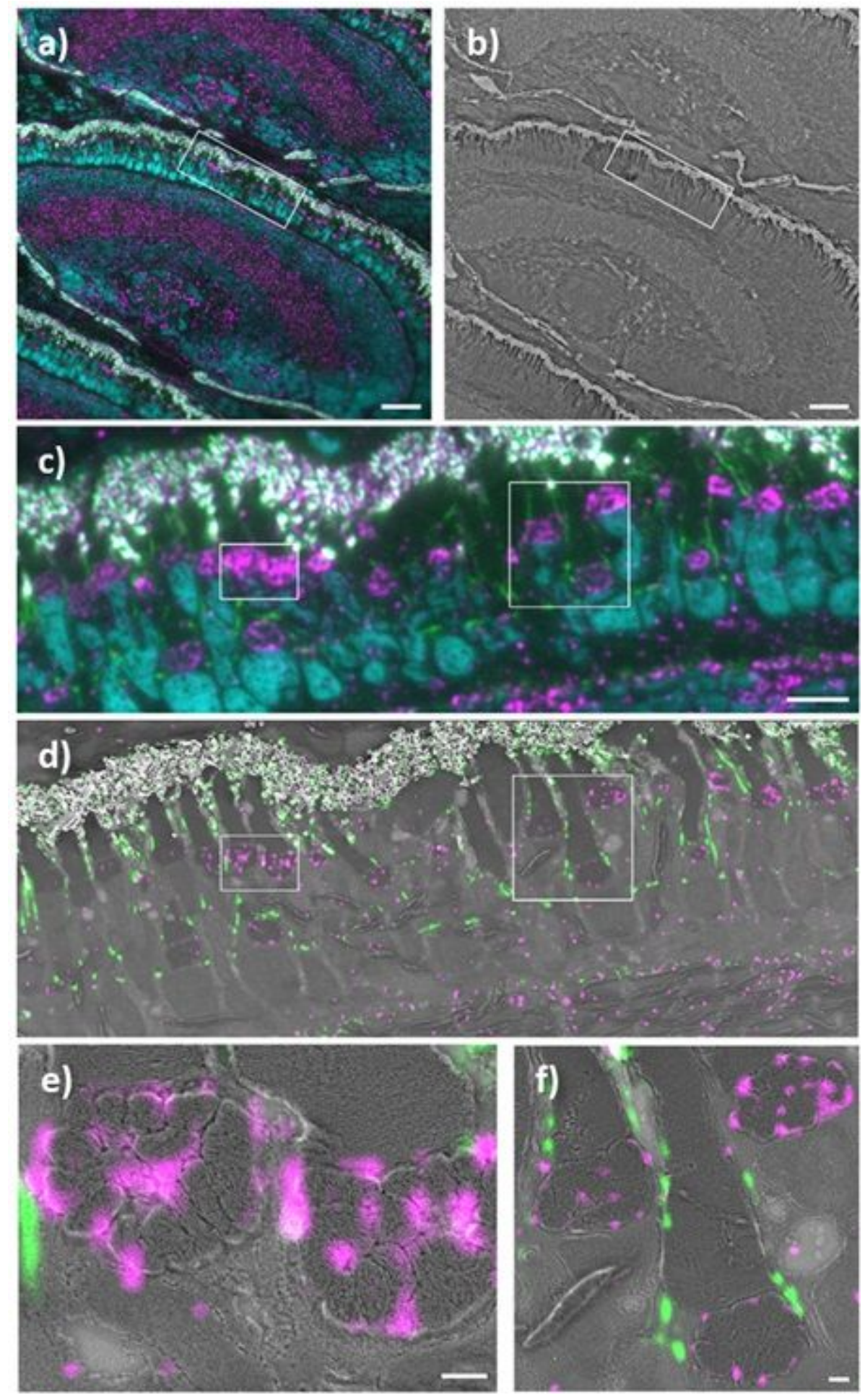

\section{Figure 5}

Chip-based CLEM imaging of a $110 \mathrm{~nm}$ thick zebrafish retina cryosections prepared by Tokuyasu method on a $600 \mu \mathrm{m}$ wide optical waveguide. (a) Diffraction-limited chip-TIRFM image. In magenta, mitochondrial clusters immunolabeled with rabbit anti Tomm20 protein (primary antibody) and Alexa Fluor 647-conjugated donkey anti-rabbit (secondary antibody). In green, actin segments labeled with Texas Red-X Phalloidin. In cyan, nuclei labeled with Sytox Green. (b) Scanning electron microscope image of the same reaion shown in (a) scanned at $30 \mathrm{~nm}$ pixel size. (c) high magnification image of the white Loading [MathJax]/jax/output/CommonHTML/jax.js 
frame in (a) showing the diffraction-limited chip-TIRFM signal of mitochondria, actin, and nuclei. (d) CLEM image of areas in frames (a) and (b). Scanning electron microscope image acquired at $4 \mathrm{~nm}$ pixel size correlates with the MUSICAL images of mitochondria (magenta) and actin (green). (e) CLEM image of the white region in (d). MUSICAL image of the Tomm20 signal (magenta) in the outer membrane of mitochondria correlating with the morphology of the complex clusters of mitochondria. The tightly packed membranes of the outer segment are clearly recognized. (f) CLEM image of MUSICAL-processed actin signal along with the outer segments (green) and three mitochondria clusters. The MUSICAL signal in (d), (e) and (f) were gamma-corrected to increase the contrast of the actin $(\mathrm{Y}=1.2)$ and the Tomm20 signal $(y=1.1)$. Scale bars $(a, b) 20 \mu m$, (c,d) $5 \mu \mathrm{m},(e, f) 500 \mathrm{~nm}$.

\section{Supplementary Files}

This is a list of supplementary files associated with this preprint. Click to download.

- SupplementaryInformation.docx 